\title{
Asymmetric Addition Catalyzed by Optically Active Polymers. IV*. Addition of Lauryl Mercaptan to Dimethyl Maleate and Dimethyl Fumarate
}

\author{
Shohei Inoue, Shin'ichi OHashi, and Yoshiro Unno \\ Department of Synthetic Chemistry, Faculty of Engineering, University of Tokyo, Tokyo, Japan.
}

(Received January 8, 1972)

\begin{abstract}
In the addition of lauryl mercaptan (LSH) to dimethyl maleate (DMM) and dimethyl fumarate(DMF), the ratio of the second-order rate constants for these geometrical isomers, $k^{\prime}{ }_{\mathrm{DMF}} / k^{\prime}{ }_{\mathrm{DMM}}$, was larger in the cases with polymeric amine and diamine as catalyst than in the cases with low-molecular-weight monoamines. In the addition reaction calalyzed by optically active amines, only the polymeric amine gave optically active product and the absolute configuration of the product obtained from DMF was opposite to that obtained from DMM.
\end{abstract}

KEY WORDS Asymmetric Addition / Dimethyl Maleate / Dimethyl

Fumarate / Polymer Catalyst / Lauryl Mercaptan / Optically Active Polymer /

In order to study the specificity of polymer catalysts mainly from the aspect of stereochemistry, we have investigated the asymmetric addition of lauryl mercaptan (LSH) to $\alpha, \beta$-unsaturated carbonyl compounds catalyzed by optically active polymeric amine ${ }^{1}$. From our previous studies, it was made clear that polymeric amine showed very specific catalytic activity. In the asymmetric addition of LSH to methacrylic esters, when the polymeric amine was used as catalyst, the effect of the ester residue on the optical yield was very marked, while the effect on the rate of the addition was not as marked. In the asymmetric addition of LSH to methyl isopropenyl ketone with polymeric amine as catalyst, the inversion in sign of $[\alpha]_{D}$ of the product was induced by only a small amount of ethanol as an additive.

In this paper, we report the results of the addition of LSH to geometrically isomeric esters, dimethyl maleate(DMM), and dimethyl fumarate(DMF), as shown in eq 1.

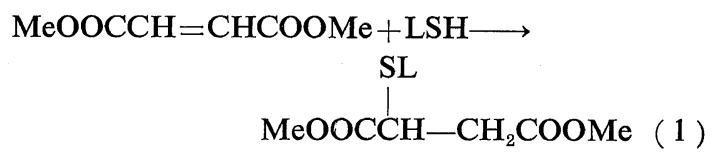

cis: Dimethyl maleate (DMM)

\footnotetext{
* For Part III, see ref 1(c).
}

trans: Dimethyl fumarate (DMF)

When the relative reactivity of the trans ester to cis ester is compared in the reactions using various amines as catalysts, it is possible to evaluate the catalytic activity of polymeric and low-molecular-weight amines, independently of the differences in the basicity and the efficiency of the catalysts. Moreover, it is very interesting to observe the effect of the geometrically isomeric structure of the unsaturated esters on the asymmetric addition with optically active amines as catalyst. Such a study is also interesting in view of the high specificity of some enzymes, such as fumarase, towards geometrically isomeric substrates.

\section{EXPERIMENTAL}

\section{Reagent}

Optically active amines except poly ( $S$-isopropylethyleneimine $)(S$-PIPI) were prepared in the same manner as those reported in the previous paper ${ }^{1}$.

$\operatorname{Poly}(S-$ and racemic-isopropylethyleneimine) were prepared starting from valine, as in the case of $\operatorname{poly}(S \text {-isobutylethyleneimine })^{1}$.

The dimethyl maleate used was a commercial product, but was purified by distillation under reduced pressure, bp $73^{\circ} \mathrm{C}(5 \mathrm{~mm})$. 
Dimethyl fumarate was purified by recrystallization from chlorofrom-diethyl ether, $\mathrm{mp}$ $106.0 \sim 106.9^{\circ} \mathrm{C}$.

$n$-Butylamine, diethylamine, triethylamine, and ethylenediamine were used after drying with calcium hydride followed by distillation.

\section{Measurement of the Rate of Addition}

Dimethyl maleate(DMM) or dimethyl fumarate(DMF) placed in a nitrogen-filled reaction vessel was dissolved in chloroform and kept at $35^{\circ} \mathrm{C}$. The reaction was initiated by adding lauryl mercaptan(LSH) with amine kept at $35^{\circ} \mathrm{C}$ to the above-mentioned solution. A fraction of the reaction mixture was taken out at regular time intervals, diluted with ethanol containing a small amount of acetic acid and titrated with $1 / 10 N$-iodine solution to determine the residual LSH concentration.

Measurement of the Extent of the Isomerization of Dimethyl Maleate to Dimethyl Fumarate

The extent of the isomerization of DMM to DMF catalyzed by amine was measured both in the presence and in the absence of LSH in coloroform at $35^{\circ} \mathrm{C}$. A fraction of the reaction mixture was taken out at regular time intervals, and the ratio of DMF to DMM measured by the use of gas chromatography.

\section{Asymmetric Addition}

The reaction was carried out in a similar manner to that described in Measrement of the Rate of Addition. When the reaction had proceeded to an almost quantitative conversion (usually after one week), solvent and unreacted materials were removed by evaporation under reduced pressure. The product was purified by recrystallization from ethanol at $-15^{\circ} \mathrm{C}$ until it showed a constant optical activity, to give dimethyl $\alpha$-dodecylthiosuccinate, $\mathrm{mp} 27.5 \sim 28.0^{\circ} \mathrm{C}$ (uncorrected).

Anal. Calcd for $\mathrm{C}_{18} \mathrm{H}_{34} \mathrm{O}_{4} \mathrm{~S}$ (346.58): C, 62.38; H, 9.91; S, 9.25. Found: $\mathrm{C}, 62.65 ; \mathrm{H}, 9.74 ; \mathrm{S}$, 9.48 .

Measurement of Optical Activity of the Products

Optical rotation at $589 \mathrm{~m} \mu$ of the products was measured in acetone solution $(c a .10 \mathrm{~g} / \mathrm{d} l)$ using a model JASCO ORD/UV-5 polarimeter.

\section{RESULTS AND DISCUSSION}

Rate of Addition of Lauryl Mercaptan to Dimethyl Maleate and Dimethyl Fumarate

The rates of the addition of lauryl mercaptan (LSH) to dimethyl maleate(DMM) and dimethyl fumarate(DMF) catalyzed by various amines were measured in chloroform at $35^{\circ} \mathrm{C}$. Figure 1 shows the relation between the reaction time and the residual LSH concentration in the ad-

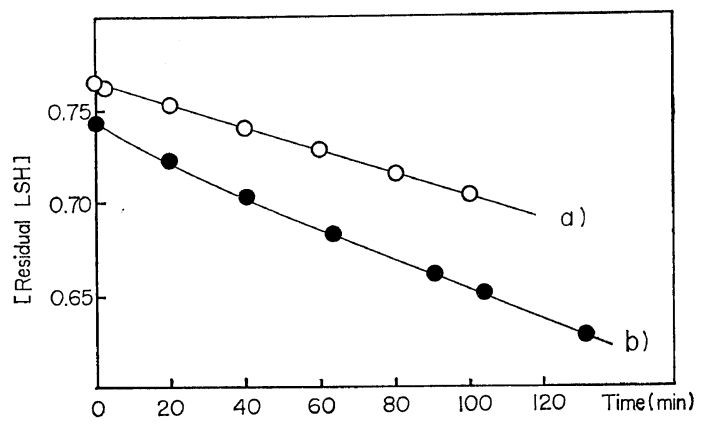

Figure 1. Addition of lauryl mercaptan to dimethyl maleate and dimethyl fumarate:

(a) $\mathrm{LSH}+\mathrm{DMM},[\mathrm{LSH}]_{0}=[\mathrm{DMM}]_{0}=0.74 \mathrm{~mol} / l,[\mathrm{~N}] /$ $[\mathrm{LSH}]_{0}=6.7 \times 10^{-2} ; \quad$ (b) $\mathrm{LSH}+\mathrm{DMF}, \quad[\mathrm{LSH}]_{0}=$ $[\mathrm{DMF}]_{0}=0.76 \mathrm{~mol} / l,[\mathrm{~N}] /[\mathrm{LSH}]_{0}=6.2 \times 10^{-2}$, catalyst, $\mathrm{Et}_{2} \mathrm{NH}$ in $\mathrm{CHCl}_{3}$ at $35^{\circ} \mathrm{C}$.

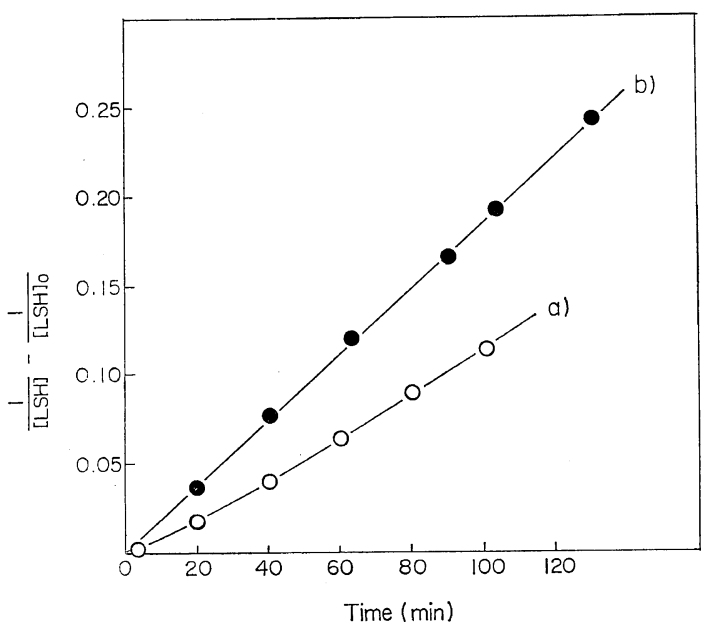

Figure 2. Second-order plots for addition of lauryl mercaptan to dimethyl maleate and dimethyl fumarate: (a) $\mathrm{LSH}+\mathrm{DMM},[\mathrm{LSH}]_{0}=[\mathrm{DMM}]_{0}=$ $0.74 \mathrm{~mol} / l,[\mathrm{~N}] /[\mathrm{LSH}]_{0}=6.7 \times 10^{-2}$; (b) $\mathrm{LSH}+\mathrm{DMF}$, $[\mathrm{LSH}]_{0}=[\mathrm{DMF}]_{0}=0.76 \mathrm{~mol} / l, \quad[\mathrm{~N}] /[\mathrm{LSH}]_{0}=6.2 \times$ 10-2; catalyst, $\mathrm{Et}_{2} \mathrm{NH}$ in $\mathrm{CHCl}_{3}$ at $35^{\circ} \mathrm{C}$. 


\section{Asymmetric Addition by Optically Active Polymer}

dition of LSH to DMM and DMF catalyzed by diethylamine (DEA). Figure 2 shows the second-order plot for the above reactions. The results indicate that this reaction is of first order each in LSH and in ester. In the cases of other amines, the reaction was also of second order. Using the second-order rate constant $(k)$ obtained, the reactivity per one nitrogen atom in the amines, $k^{\prime}$, was calculated assuming that all nitrogen atoms in the amines may act as catalytic species. The value of $k^{\prime}$ is shown in Table $I$.

Table I. Addition of lauryl mercaptan to dimethyl maleate and dimethyl fumarate

\begin{tabular}{|c|c|c|c|c|}
\hline Catalyst & $k^{\prime} \mathrm{DMF}^{\mathrm{b}}$ & $k^{\prime}{ }_{\mathrm{DMM}}^{\mathrm{b}}$ & $R^{\mathrm{c}}$ & $R^{\prime \mathrm{d}}$ \\
\hline \multicolumn{5}{|l|}{$i^{-} \mathrm{Bu}$} \\
\hline$\underset{i-\mathrm{Pr}}{-\left(-\mathrm{CH}-\mathrm{CH}_{2}-\mathrm{NH}-\right)_{-n}^{-n}}$ & $\begin{array}{l}0.104 \\
0.070\end{array}$ & $\begin{array}{l}0.031 \\
0.017\end{array}$ & $\begin{array}{l}3.4 \\
4.1\end{array}$ & \\
\hline$-\left(-\mathrm{CH}-\mathrm{CH}_{2}-\mathrm{NH}-\right)_{-n} \mathrm{RS}^{\mathrm{e}}$ & 0.17 & 0.038 & 4.5 & \\
\hline $\mathrm{EtNH}-\mathrm{CH}-\mathrm{CH}_{2} \mathrm{NHBu}$ & 0.50 & 0.13 & 4.0 & 4.8 \\
\hline$n-\mathrm{BuNH}_{2}$ & 0.097 & 0.067 & 1.4 & 1.6 \\
\hline $\mathrm{Et}_{2} \mathrm{NH}$ & 0.38 & 0.22 & 1.8 & 3.4 \\
\hline $\mathrm{Et}_{3} \mathrm{~N}$ & 0.062 & 0.021 & 3.0 & 3.2 \\
\hline $\mathrm{H}_{2} \mathrm{NCH}_{2} \mathrm{CH}_{2} \mathrm{NH}_{2}$ & 0.093 & 0.047 & 2.0 & \\
\hline $\mathrm{Ph}$ & 0.014 & 0.0079 & 1.8 & \\
\hline $\mathrm{H}_{2} \mathrm{~N}-\mathrm{CH}-\mathrm{CH}_{3} \mathrm{RS}^{\mathrm{e}}$ & 0.016 & 0.0082 & 2.0 & \\
\hline
\end{tabular}

a Reaction in $\mathrm{CHCl}_{3}$ at $35^{\circ} \mathrm{C}$.

b $k^{\prime}=k /[N]\left(l^{2} / \mathrm{mol}^{2} \mathrm{~min}\right) ; k$, observed second-order rate constant $(l / \mathrm{mol} \mathrm{min})$.

c $R=k^{\prime}{ }_{\text {DMF }} / k^{\prime}{ }_{\text {DMM }}$.

d $R^{\prime}$ is the corrected value of $R$ for isomerization.

- Absolute configuration.

$k^{\prime}$ is much different from each other depending on the structure of the amines. No simple correlation was observed between $k^{\prime}$ and the structure of the amines. The variation in $k^{\prime}$ values is considered to be at least partly due to the difference in the basicity and the catalytic efficiency of these amines.

Next, the relative reactivity of trans ester (DMF) to cis ester (DMM), $R=k_{\mathrm{DMF}}^{\prime} / k_{\mathrm{DMM}}^{\prime}$, was calculated and is also shown in Table I. As seen in the Table, $R$ is larger in the cases with polymeric amine, poly $(S$-isobutylethyleneimine $)$ (PIEI) and with diamine, $S$-1- $n$-butylamino-2-
ethylamino-4-methylpentane(BEMP) than in the cases with other low-molecular-weight amines.

\section{Isomerization of Dimethyl Maleate to Dimethyl Fumarate}

It is well known that the isomerization of DMM to DMF is catalyzed by amines. ${ }^{2}$ It has been proposed that the isomerization is due to the nucleophilic attack of $\mathrm{N}$ to carbon-carbon double bond followed by the rotation of the carbon-carbon bond ${ }^{3}$. In the present system, if isomerization occurs appreciably, $k_{\text {DMM }}^{\prime}$ must be corrected for the isomerization. For this reason, the extent of the isomerization of DMM to DMF catalyzed by amines was measured in the presence of $\mathrm{LSH}$ in chloroform at $35^{\circ} \mathrm{C}$. Figure 3 shows the appearance of DMF in DMM-Amine system. The figure shows that when DEA and $n$-butylamine (BA) were used as catalyst, the rate of the appearance of DMF was very fast while in the cases of other amines such as PIEI, BEMP, and triethylamine (TEM) it

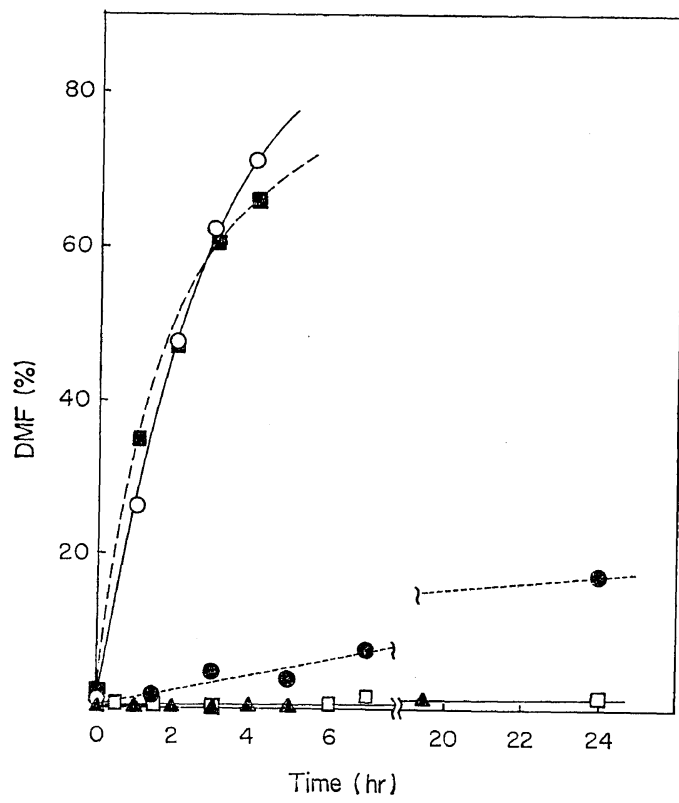

Figure 3. Isomerization of dimethyl maleate to dimethyl fumarate catalyzed by amine in the absence of lauryl mercaptan:

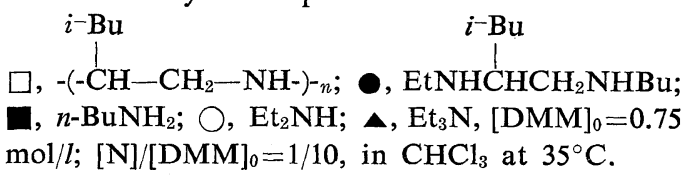


was very slow. It is well known that tertiary amines rear range DMM to DMF very slowly owing to their high steric requirements ${ }^{3}$. In the cases of BEMP and PIEI, although these are secondary amines, the very bulky substituent on the nitrogen is considered to decrease the extent of isomerization. Besides this, two pairs of hydrogen bonding between NH of PIEI or BEMP and the ester seem to prevent the rotation of the carbon-carbon bond even if the $\mathrm{N}$ attacks the double bond, contributing to the retention of the cis structure.

Figure 4 shows the isomerization of DMM to DMF catalyzed by amines in the presence of LSH. In this case also, DEA and BA induced very fast isomerization while PIEI, BEMP, and TEA induced very slow one. Moreover, in this

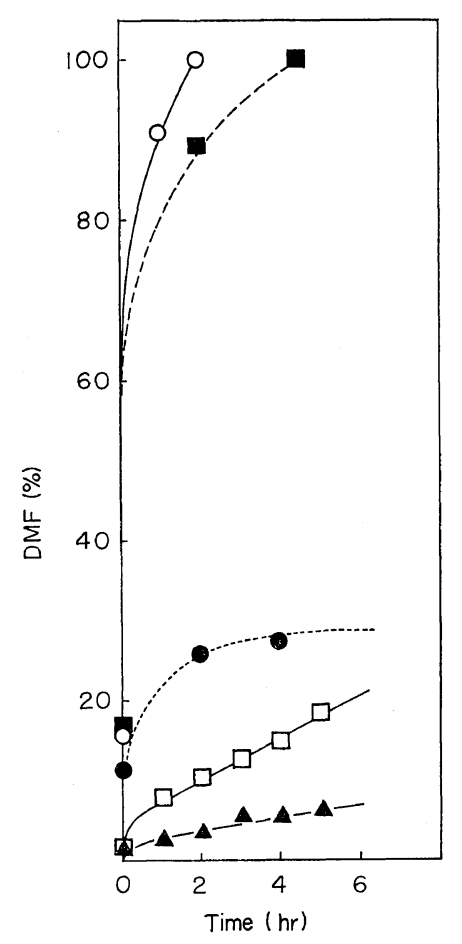

Figure 4. Isomerization of dimethyl maleate to dimethyl fumarate catalyzed by amine in the presence of lauryl mercaptan:

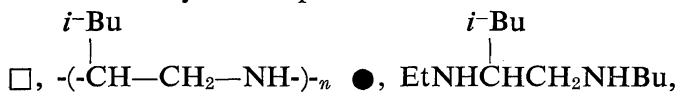

$$
\begin{aligned}
& \boldsymbol{\square}, n-\mathrm{BuNH}_{2}, \quad \bigcirc, \mathrm{Et}_{2} \mathrm{NH}, \quad \boldsymbol{\Delta}, \mathrm{Et}_{3} \mathrm{~N} \text {; } \\
& {[\mathrm{DMM}]_{0}=[\mathrm{LSH}]_{0}=0.75 \mathrm{~mol} / l ;[\mathrm{N}]_{0} /[\mathrm{DMM}]_{0}=1 / 10 \text {, }} \\
& \text { in } \mathrm{CHCl}_{3} \text { at } 35^{\circ} \mathrm{C} \text {. }
\end{aligned}
$$

case the isomerization was faster than that in the case without LSH. On the other hand, when DMM was added to LSH in chloroform in the absence of amine, only a small amount of DMF appeared, but the amount did not increase. Therefore in the LSH-DMM-Amine system, $\mathbf{L S} \ominus$ as well as $\mathrm{N}$ is considered to act as a nucleophile to result in the faster isomerization than in the case without LSH.

In any event, $k_{\text {DMM }}^{\prime}$ must be corrected for isomerization, although it is very slow in the cases of PIEI and BEMP. The ratio of DMF to DMM was measured under the same condition as that used for the measurement of the reaction rate and $k_{\text {DMM }}^{\prime}$ was corrected according to eq 2 ;

$$
k_{\mathrm{DMM}}^{\mathrm{App}}=\alpha k_{\mathrm{DMF}}^{\prime}+(1-\alpha) k_{\mathrm{DMM}}^{\prime \mathrm{Net}}
$$

where $k_{\mathrm{DMM}}^{\mathrm{Net}}$ is the corrected rate constant for DMM, $k_{\mathrm{DMM}}^{\mathrm{App}}$ is the apparent rate constant observed, and $\alpha$ is the fraction of DMF in the reaction mixture. Using $k_{\mathrm{DMM}}^{\mathrm{Net}}, R$ is corrected and the value, $R^{\prime}$, is shown in Table I. Even after this correction, $R^{\prime}$ is larger in the cases with PIEI and BEMP than in the cases with other low-molecular-weight amines. In other words, PIEI or BEMP is more specific towards the cis ester than other low-molecular-weight amines. The larger $R^{\prime}$ value may by related to the presence of two pairs of hydrogen bonding between NH and ester group possible in PIEI or BEMP, which results in the difference in the extent of interaction of the ester with the catalyst or the reactivity of cis or trans ester.

Asymmetric Addition of Lauryl Mercaptan to Dimethyl Maleate and Dimethyl Fumarate

It is very interesting to study the effect of the difference in the geometrically isomeric structure of the esters on the asymmetric addition. By this method it is possible to know the mode of the interaction between the catalyst and the ester. The asymmetric addition of LSH to DMM and DMF was investigated in chloroform with PIEI, BEMP, and $R$ - $\alpha$-phenylethylamine as catalyst. The results are shown in Table II.

As Table II shows, when PIEI was used as catalyst, optically active products were obtained. On the other hand, low-molecular-weight amines 
Table II. Asymmetric addition of lauryl mercaptan to dimethyl maleate and dimethyl fumarate ${ }^{a}\left([\alpha]_{D}\right.$ of the product $\left.{ }^{\mathrm{b}}\right)$

\begin{tabular}{|c|c|c|c|}
\hline \multirow[b]{2}{*}{ Catalyst } & & \multicolumn{2}{|c|}{ Ester } \\
\hline & & $\underset{\mathbf{H}}{\mathrm{MeOOC}} \underset{(\mathrm{DMM})}{\mathrm{C}=\mathrm{C}}\langle\underset{\mathrm{H}}{\mathrm{COOMe}}$ & $\underset{\mathbf{H}}{\mathrm{MeOOC}} \underset{(\mathrm{DMF})}{\mathrm{C}=\mathrm{C}} \underset{\mathrm{COOMe}}{\langle\mathbf{H}}\langle\underset{\mathrm{COO}}{\mathrm{M}}$ \\
\hline$i_{-} \mathrm{Bu}$ & & & \\
\hline$-\left(-\mathrm{CH}-\underset{\text { (PIEI) }}{\left.\mathrm{CH}_{2}-\mathrm{NH}-\right)_{-n}}\right.$ & $(\mathbf{S})^{\mathrm{c}}$ & -1.55 & +1.49 \\
\hline $\mathrm{Ph}$ & & & \\
\hline $\begin{array}{c}\text { EtNHCHCH} \mathrm{HCH}_{2} \mathrm{NHBu} \\
(\mathrm{BEMP}) \\
\mathrm{Ph}\end{array}$ & $(\mathbf{S})^{\mathrm{e}}$ & 0 & 0 \\
\hline $\mathrm{H}_{2} \mathrm{~N}-\mathrm{CH}-\mathrm{CH}_{3}$ & $(\mathrm{R})^{\mathrm{c}}$ & 0 & 0 \\
\hline
\end{tabular}

a Reacion in $\mathrm{CHCl}_{3}$ at $35^{\circ} \mathrm{C}$. $[\mathrm{N}] /[\mathrm{LSH}]_{0}=1 / 10$, [Ester $]_{0} /[\mathrm{LSH}]_{0}=1.0$

b Measured in acetone $(c=10 \mathrm{~g} / \mathrm{d} l)$ at room temperature.

- Absolute configuration.

gave only optically inactive products. Moreover, when PIEI was used, it is very interesting that the sign of $[\alpha]_{D}$ of the products obtained from DMF and DMM was opposite to each other.

As we have discussed before, the reaction is considered to proceed as follows

$$
\mathrm{LSH}+\mathrm{N} \leftrightharpoons \mathrm{LS} \ominus_{+} \stackrel{\mathrm{NH}}{\bigvee}
$$

$$
\begin{aligned}
& \mathrm{LS} \odot \oplus \mathrm{NH}+\mathrm{MeOOCCH}=\mathrm{CHCOOMe} \longrightarrow
\end{aligned}
$$

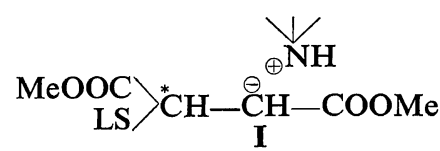

$$
\begin{aligned}
& \stackrel{\mathrm{I}^{+}}{\longrightarrow} \underset{\mathrm{LS}}{\stackrel{\mathrm{MeOOC}}{\longrightarrow}} \stackrel{*}{\mathrm{CH}}-\underset{\mathbf{I I}}{\mathrm{CH}_{2}-\mathrm{COOMe}}
\end{aligned}
$$

The asymmetric carbon is formed at step (4). The formation of optically active products indicates that the direction of the addition of LS $\ominus$ to the ester is controlled in the case with PIEI. Hydrogen bonding between NH of the catalyst and the ester group is considered, as the most important factor which controls the direction. $[\alpha]_{\mathrm{D}}$ of the products obtained from DMM or DMF is much larger compared with that obtained from methyl crotonate( $\mathrm{MCr}$ ), benzalacetone(BA)and benzalacetophenone(BAP), where asymmetric carbon is formed at step (4). $\left([\alpha]_{\mathrm{D}}\right.$ of the product obtained from $\mathrm{MCr}, \mathrm{BA}$, and BAP was $+0.19,-0.04$, and -0.08 respectively, using PIEI as catalyst.) Considering this fact, two pairs of hydrogen bonding between $\mathrm{NH}$ of the catalyst and ester group are considered to be effective for efficient steric control.

In the case of DMF, the formation of the optically active product or the preferential formation of one of the enantiomers indicates that LS $\ominus$ adds to DMF preferentially from one side (a or $b$ in Figure 5) of the plane involving the double bond of DMF. On the other hand, in the case of DMM, the observed asymmetric synthesis suggests that $\mathrm{LS} \ominus$ not only prefers one side of the plane of the double bond but also reacts selectively with one of the two carbon atoms binding the double bond $\left(\mathrm{C}_{\mathrm{A}}\right.$ or $\mathrm{C}_{\mathrm{B}}$ in Figure 5).

Such a control of the direction from which $\mathrm{LS} \ominus$ adds to the ester is considered to be made possible by hydrogen bonding between $\mathrm{NH}$ and the ester group and by the steric interaction

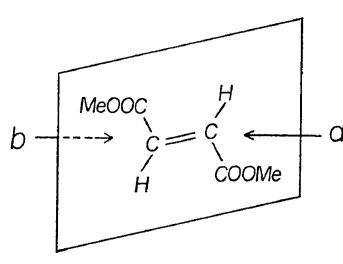

Dimethyl Fumarate

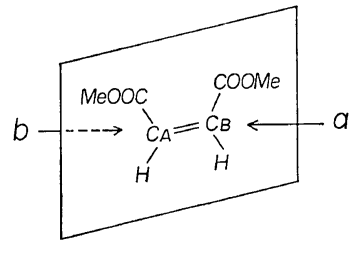

Dimethyl Maleate
Figure 5. 
between the ester residue and the substituent of the polymeric amine with the same absolute configuration. In the cases of low-molecularweight amines, the control of the direction of the addition by such a mechanism is considered very weak, if at all.

Thus, the polymeric catalyst PIEI forms hydrogen bond with the ester and makes it possible for $\operatorname{LS} \ominus$ to add to the ester preferentially from one direction. In other words, PIEI may be said to have an ability to distinguish not only the enantiotopic face of the double bond but also the two carbon atoms of the double bond.
Acknowledgement. The authors are grateful to Professor Teiji Tsuruta for valuable suggestions and comments.

\section{REFERENCES}

1. (a) S. Inoue, S. Ohashi, A. Tabata, and T. Tsuruta, Makromol. Chem., 112, 66 (1968); (b) S. Ohashi and S. Inoue, ibid., 150, 105 (1971); (c) S. Ohashi and S. Inoue, ibid., in press.

2. G.R. Clemo and S.B. Graham, J. Chem. Soc., 1930, 213.

3. L. Grünbaum, S. Patai, and Z. Rappoport, $J$. Chem. Soc., Sect. B, 1966, 1133. 